\title{
Genome sequencing and analysis of the first complete genome of Lactobacillus kunkeei strain MP2, an Apis mellifera gut isolate
}

Freddy Asenjo, Alejandro Olmos, Patricia Henríquez-Piskulich, Victor Polanco, Patricia Aldea, Juan A Ugalde, Annette N Trombert

Background. Honey bee (Apis mellifera) is the most important pollinator in agriculture worldwide. However, the number of honey bees has fallen significantly since 2006, becoming a huge ecological problem nowadays. The principal cause is CCD or Colony Collapsed Disorder, characterized by the seemingly spontaneous abandonment of hives by their workers. One of the characteristics of CCD in honey bees is the alteration of the bacterial communities in their gastrointestinal tract, mainly due to the decrease of Firmicutes populations, such as the Lactobacilli. At this time, the causes of these alterations remain unknown. We recently isolated a strain of Lactobacillus kunkeei ( $L$. kunkeei strain MP2) from the gut of Chilean honey bees. L. kunkeei, is one of the most commonly isolated bacterium from the honey bee gut and is highly versatile in different ecological niches. In this study, we aimed to elucidate in detail, the L. kunkeei genetic background and perform a comparative genome analysis with other Lactobacillus species. Methods. L. kunkeei MP2 was originally isolated from the guts of Chilean A. mellifera individuals. Genome sequencing was done using Pacific Biosciences single-molecule realtime sequencing technology. De novo assembly was performed using Celera assembler. The genome was annotated using Prokka, and functional information was added using the EggNOG 3.1 database. In addition, genomic islands were predicted using IslandViewer, and pro-phage sequences using PHAST. Comparisons between $L$. kunkeei MP2 with other $L$. kunkeei, and Lactobacillus strains were done using Roary. Results. The complete genome of $L$. kunkeei MP2 comprises one circular chromosome of 1,614,522 nt. with a GC content of $36,9 \%$. Pangenome analysis with $16 \mathrm{~L}$. kunkeei strains, identified 113 unique genes, most of them related to phage insertions. A large and unique region of $L$. kunkeei MP2 genome contains several genes that encode for phage structural protein and replication components. Comparative analysis of MP2 with other Lactobacillus species, identified several unique genes of $L$. kunkeei MP2 related with metabolism, biofilm generation, survival under stress conditions, and mobile genetic elements (MGEs). Discussion. The presence of multiple mobile genetic elements, including phage sequences, suggest a high degree of genetic variability in L. kunkeei. Its versatility and ability to survive in different 
ecological niches (bee guts, flowers, fruits among others) could be given by its genetic capacity to change and adapt to different environments. L. kunkeei could be a new source of Lactobacillus with beneficial properties. Indeed, L. kunkeei MP2 could play an important role in honey bee nutrition through the synthesis of components as isoprenoids. 
1 Genome sequencing and analysis of the first complete genome of Lactobacillus

2 kunkeei strain MP2, an Apis mellifera gut isolate

3 Freddy Asenjo ${ }^{1}$, Alejandro Olmos², Patricia Henríquez-Piskulich ${ }^{3}$, Victor Polanco²,

4 Patricia Aldea ${ }^{3}$, Juan A. Ugalde ${ }^{1}$, Annette N. Trombert ${ }^{2}$

5

$6 \quad{ }^{1}$ Centro de Genética y Genómica, Facultad de Medicina, Clinica Alemana - Universidad

7 del Desarrollo, Santiago, Chile.

8 '2Centro de Genómica y Bioinformática, Facultad de Ciencias, Universidad Mayor,

9 Santiago, Chile.

$10{ }^{3}$ Centro de Estudios Apícolas CEAPI Mayor, Facultad de Ciencias, Universidad Mayor,

11 Santiago, Chile.

12

13 Corresponding Authors:

14 Juan A. Ugalde ${ }^{1}$

15 Avda. Las Condes 12438, Santiago, Chile

16 Email address: jugalde@udd.cl

17 Annette N. Trombert ${ }^{2}$

18 Camino a La Piramide 5750, Santiago, Chile

19 Email address: annette.trombert@umayor.cl

20

21

22

23 


\section{Abstract}

25 Background. Honey bee (Apis mellifera) is the most important pollinator in agriculture worldwide. However, the number of honey bees has fallen significantly since 2006,

27 becoming a huge ecological problem nowadays. The principal cause is CCD or Colony 28 Collapsed Disorder, characterized by the seemingly spontaneous abandonment of hives by their workers. One of the characteristics of CCD in honey bees is the alteration of the bacterial communities in their gastrointestinal tract, mainly due to the decrease of Firmicutes populations, such as the Lactobacilli. At this time, the causes of these alterations remain unknown.

We recently isolated a strain of Lactobacillus kunkeei (L. kunkeei strain MP2) from the gut of Chilean honey bees. L. kunkeei, is one of the most commonly isolated bacterium from the honey bee gut and is highly versatile in different ecological niches. In this study, we aimed to elucidate in detail, the L. kunkeei genetic background and perform a comparative genome analysis with other Lactobacillus species.

Methods. L. kunkeei MP2 was originally isolated from the guts of Chilean A. mellifera individuals. Genome sequencing was done using Pacific Biosciences single-molecule real-time sequencing technology. De novo assembly was performed using Celera assembler. The genome was annotated using Prokka, and functional information was added using the EggNOG 3.1 database. In addition, genomic islands were predicted using IslandViewer, and pro-phage sequences using PHAST. Comparisons between $L$. kunkeei MP2 with other L. kunkeei, and Lactobacillus strains were done using Roary.

Results. The complete genome of $L$. kunkeei MP2 comprises one circular chromosome of $1,614,522$ nt. with a GC content of $36,9 \%$. Pangenome analysis with 16 L. kunkeei 
47 strains, identified 113 unique genes, most of them related to phage insertions. A large

48 and unique region of $L$. kunkeei MP2 genome contains several genes that encode for

49 phage structural protein and replication components. Comparative analysis of MP2 with

50 other Lactobacillus species, identified several unique genes of L. kunkeei MP2 related

51 with metabolism, biofilm generation, survival under stress conditions, and mobile

52 genetic elements (MGEs).

\section{Discussion.}

54 The presence of multiple mobile genetic elements, including phage sequences, suggest

55 a high degree of genetic variability in L. kunkeei. Its versatility and ability to survive in

56 different ecological niches (bee guts, flowers, fruits among others) could be given by its

57 genetic capacity to change and adapt to different environments. L. kunkeei could be a 58 new source of Lactobacillus with beneficial properties. Indeed, L. kunkeei MP2 could 59 play an important role in honey bee nutrition through the synthesis of components as 60 isoprenoids.

\section{Introduction}

64 The honey bee (Apis mellifera) is the most important pollinator in agriculture worldwide, 65 playing a key role in the human food supply by providing pollination services for diverse 66 crops (Evans \& Schwarz, 2011). However, from 2006 to this day, an unusual decreased 67 in honey bee colonies has been taking place, known as Colony Collapse Disorder 68 (CCD). CCD describes the seemingly spontaneous abandonment of the hives by honey 69 bee workers, where queens often stay in the hive accompanied by a small group of 
70 nurse worker bees. The specific causes of CCD are unknown, but several factors can

71 impact the health of honey bees, and contribute to this phenomenon: (1) pests and

72 diseases (such as, American foulbrood, European foulbrood, chalkbrood nosema, small

73 hive beetles, and tracheal mites); (2) the use of chemicals in bee colonies, and their

74 surrounding environment; (3) beekeeping practices; (4) agricultural practices and (5)

75 climate change (Henry et al., 2012; Di Pasquale et al., 2013; Di Prisco et al., 2013).

76 Multiple studies have suggested that CCD directly affects the microbial composition of

77 the honey bee gut microbiota. Eight dominant groups can be found in the honey bee gut

78 (Cox-Foster et al., 2007; Martinson et al., 2011): Gammaproteobacteria

79 (Enterobacteriaceae and Pasteurellaceae), Betaproteobacteria (Neisseriaceae),

80 Alphaproteobacteria (Rhizobiales, Acetobacteraceae), Firmicutes (Lactobacillus sp.),

81 and Actinobacteria (Bifidobacterium sp.) groups (Cox-Foster et al., 2007; Martinson et

82 al., 2011). Gut microbiome studies from individuals obtained from colonies affected and

83 non-affected by CCD, indicated an increase in the Gammaproteobacteria, and a

84 decrease of the Firmicutes in affected colonies, showing how the CCD condition affects

85 commensal communities in the honey bee gut (Cox-Foster et al., 2007). Firmicutes

86 includes Gram-positive and low-G+C bacteria, such as the Lactobacillus genus, where

87 some of its members have been implicated in the fitness improvement of honey bees

88 (Audisio \& Benítez-Ahrendts, 2011; Audisio, Sabaté \& Benítez-Ahrendts, 2015).

89 The study of lactobacilli members of the honey bee microbiota can give us information

90 about beneficial species for honey bees. One of the most common lactobacilli species

91 present in the honey bee gut microbiota is Lactobacillus kunkeei, described for the first

92 time as a spoilage organism isolated from commercial grape wine (Edwards et al, 
93 1998). Characterized as a Gram-positive fructophilic lactic acid bacterium (FLAB), $L$. 94 kunkeei possesses a weak catalase activity and has the ability to ferment 95 carbohydrates, such as glucose, fructose, sucrose, raffinose and mannitol but prefer 96 fructose. The fermentation products of these reactions are lactic acid and acetic acid 97 (Edwards et al., 1998; Bae, Fleet \& Heard, 2006; Endo, Futagawa-Endo \& Dicks, 2009; 98 Endo, 2012). L. kunkeei can be found in fructose rich-niches, including honey, 99 beebread, wine and flowers (Vásquez et al., 2012; Endo et al., 2012). Furthermor, it is 100 present in the gastrointestinal tract of several insects found in flowers, such as tropical 101 fruit flies, Camponotus spp (carpenter ants), bumblebees and honey bees (Neveling, 102 Endo \& Dicks, 2012; Anderson et al., 2013; Endo \& Salminen, 2013). During the 103 summer months, L. kunkeei is the most frequent lactobacilli isolate from the honey bee 104 gut (Corby-Harris, Maes \& Anderson, 2014; McFrederick et al., 2014).

105 In previous work, we isolated a L. kunkeei strain (named as MP2) from the gut of 106 Chilean honey bees from María Pinto, Melipilla (Olmos et al., 2014). The genome of this 107 strain was sequenced using the Illumina MiSeq platform, which resulted in a draft 108 genome of 44 contigs, for a total genome size of 1,581,395 bp, and 826 well-annotated 109 protein coding-genes (Olmos et al., 2014). The nature of the short-reads used for this 110 assembly, did not allow to completely resolve the genome without gaps. In addition, 111 multiple repetitions, including the presence of multiple copies of the ribosomal operon, 112 could not be resolved in this draft genome. To overcome these limitations, we 113 performed a re-sequencing of the L. kunkeei MP2 genome, using single molecule 114 sequencing in the Pacific Biosciences platform. 
115 In this work, we report the first complete genome sequence of $L$. kunkeei MP2, its

116 characterization, and comparison with other Lactobacillus genomes.

\section{Methods}

119 DNA isolation and genome sequencing

120 The L. kunkeei strain MP2 reported in this study was isolated in a previous study from

121 the gut of a honey bee (Apis mellifera), collected from commune hives in the Maria

122 Pinto area, Melipilla Province in the Central zone of Chile (Olmos et al., 2014).

123 Collection and use of honey bees for these studies was reviewed and approved by the

124 Bioethics Committee of Universidad Mayor, which is governed by the regulations of the 125 Animal Health Services of Chile.

126 For DNA extraction, L. kunkeei MP2 colonies were cultured in MRS broth $\left(37^{\circ} \mathrm{C}, 5 \%\right.$

$127 \mathrm{CO}_{2}$ ) and genomic DNA was obtained using a silica-based protocol (Boom et al., 1999).

128 Briefly, bacterial pellet was lysed using a solution composed of SDS $10 \%$, proteinase $\mathrm{K}$ 129 (10 mg/mL, Thermo Scientific) and lysozyme (5 mg/mL, Pierce) at $37^{\circ} \mathrm{C}$ for $60 \mathrm{~min}$. The 130 lysate was mixed with guanidine chloride $6 \mathrm{M}$ and a silica suspension (50\% w/v) and 131 incubated for $10 \mathrm{~min}$. The silica was centrifuged, and DNA was recovered, after washes 132 with $70 \%$ alcohol, into sterile, free nuclease water. Approximately $13.5 \mu \mathrm{g}$ of DNA were

133 used to construct sequencing libraries with an average insert size of $20 \mathrm{~kb}$, and 134 sequenced using one SMRT cell (P6-C4 Chemistry) on a PacBio RSIl sequencer 135 (Pacific Biosciences) at the UCSD IGM Genomics Center.

136 Genome assembly and annotation 
137 Raw reads ( $\sim$ Gbps) were processed to remove SMRT bell adapters, short and low138 quality reads ( $<80 \%$ accuracy) using SMRT Analysis version 2.3. A total of 154,044

139 filtered reads (average length, 9Kb) were used for de novo assembly using Celera 140 Assembler version 8.3 (Myers et al., 2000), with self-correction of the PacBio reads 141 (Berlin et al., 2015). Polishing was done using Quiver, using SMRT Analysis version 142 2.3. Comparisons between the previously sequenced L. kunkeei MP2 genome 143 (Accession number PRJNA257367) (Olmos et al., 2014), as well with the other two 144 available genome sequences (Porcellato et al., 2015; Djukic et al., 2015) were 145 performed using MUMMER (Kurtz et al., 2004). Genome annotation was performed 146 using Prokka version 1.11 (Seemann, 2014). The predicted CDS were classified into 147 EggNOG categories using HMMER version 3.1 (http://hmmer.org) against the EggNOG 148 4.1 database (Powell et al., 2014) with an E-value cutoff of 1E-05. Genomic islands 149 were annotated using IslandViewer 3 (Dhillon et al., 2015), and possible phage 150 sequences were searched using PHAST (Zhou et al., 2011). Genome visualization was 151 done using Circos version 0.69 (Krzywinski et al., 2009). The genome sequence an 152 assembly is available at NCBI with the accession number PRJNA298292.

\section{Pan-genome analysis}

154 Comparative genomic analysis was performed from a list of selected genomes from 155 Lactobacillus species, as well as other strains of $L$. kunkeei (Table 1). To avoid possible 156 biases in the comparisons due to different annotation procedures, all of the genomes 157 were re-annotated using Prokka version 1.11 (Seemann, 2014). Comparisons between 158 all the selected genomes, were done using Roary (Page et al., 2015), with a blast 159 identity cutoff of $97 \%$ for the comparison between L. kunkeei strains, and a $40 \%$ for the 
160 comparison between Lactobacillus species. In addition at the genus level, paralog

161 splitting was disabled. Visualization of the pan-genome data was done using Anvi'o

162 (Eren et al., 2015).

163 Phylogenetic analysis

164 16S rRNA gene sequences were obtained from the Silva database (Quast et al., 2013),

165 and aligned using the SINA webserver aligner (Pruesse, Peplies \& Glöckner, 2012) with

166 default parameters, and using the Bacteria variability profile. For the L. kunkeei strain

167 tree, we used the core genome (genes shared by all L. kunkeei strains), aligning the

168 genes using Prank (Löytynoja, 2014). For both cases, the phylogenetic trees were

169 generated using FastTree v 2.1.7 (Price, Dehal \& Arkin, 2010), with the -slow option.

170 Results and Discussion

171 Assembly description

172 The PacBio reads obtained for $L$. kunkeei MP2 were assembled using MHAP (Berlin et

173 al., 2015) implemented in the PBcR pipeline (Celera Assembler 8.3) (Myers et al.,

174 2000). This de novo assembly resulted in one contig, representing the complete

175 genome of $L$. kunkeei MP2 in a single 1,614,522 nt chromosome. A total of 1,468 CDS

176 were predicted in the genome, 67 tRNA and 5 copies of the ribosomal operon.

177 Functional annotation was done using EggNOG V 4.1 (Powell et al., 2014), and the 178 summary of functional categories is shown on Table 2 (gene annotation on

179 Supplementary Table 1). The \%GC content of the genome was $36.9 \%$, and several 180 features of interests, such as the presence of prophage regions, were found. With this

181 assembly, we were able to differentiate the three ribosomal operons that are present in 
182 the chromosome, something that was not possible in the previous sequenced genome

183 of this strain (Olmos et al., 2014).

184 A comparison of the assembly of L. kunkeei MP2 obtained in this work, with the 185 previously obtained using Illumina sequencing (Olmos et al., 2014), is shown on Figure 186 1. All of the previous assembled contigs mapped to the current assembly, and several 187 gaps on the sequence were completed in this new version of the genome.

188

189 Central Metabolism of L. kunkeei MP2

190 Energy metabolism. MP2 has the complete route for acetate synthesis, with the 191 presence of the gene codifying for phosphoglycerate kinase. No genes codifying for 192 phosphoribulokinase (PRK) and ribulose-biphosphate carboxylase (RbcL), two of the 193 enzymes involved in the synthesis of glyceraldehyde-3-phosphate synthesis, were 194 found on the genome.

195 Carbohydrate metabolism. The genes that encode for the enzymes 196 phosphofructokinase/glucokinase (PFK) and Fructose-biphosphate aldolase (FBA), 197 were not found in the genome of L. kunkeei MP2. These enzymes are part of the 198 Embden-Meyerhof pathway and are involved in the homofermentative metabolism of 199 lactic acid. As a fructophilic bacterium, L. kunkeei MP2 can synthesize ribose-5200 phosphate through pentose phosphate pathway from fructose and obtain PRPP 201 (phosphoribose pyrophosphate), the precursor of purine, pyrimidine and histidine 202 metabolism. For the synthesis of ribose-5-phosphate, L. kunkeei uses the route from B203 D-fructose-6-phosphate through D-arabino-Hex-3-ulose-6-phosphate intermediate. $L$. 204 kunkeei MP2 can synthesize UDP-glucose and has two isoprenoid biosynthesis 
205 pathways, the mevalonate and the non-mevalonate pathways. Isoprenoids include 206 carotenoids, sterols, prenyl side-chains of chlorophylls, and plastoquinone, exhibiting 207 many biological functions (Daum et al., 2009). In whiteflies (Bemisia tabaci), the 208 genome of its endosymbiotic bacteria, Candidatus Portiera aleyrodidarum, encodes for 209 key enzymes in carotenoids synthesis, suggesting that whitefly not only can acquire 210 carotenoids from the diet, but also from their microbiota (Sloan \& Moran, 2012).

211 Therefore, if L. kunkeei produces key enzymes involved in isoprenoid synthesis, it is 212 possible that it could be playing an important role in honey bee nutrition.

213 Nucleotide and amino acid metabolism. The pathways for purine biosynthesis are 214 complete. However, in the de novo pirimidine pathway, L. kunkeei lacks the gene pyrB, 215 which codifies for the aspartate carbamoyl transferase, and ndk, codifying for the 216 nucleoside diphosphate kinase. The analysis of the metabolic pathways in MP2 217 revealed a minimal amino acid auxotrophy (methionine or cysteine), with the presence 218 of the genes that encode for a D-methionine transport system, suggesting the ability of 219 L. kunkeei MP2 to acquire methionine/cysteine from the environment. These results are 220 in line with previous reports of the lactobacilli being auxotrophic for both methionine and 221 cysteine (Seefeldt \& Weimer, 2000), and where the supplement of culture media with 222 these amino acids improved bacterial growth (Lozo et al., 2008). A gene that encodes 223 for serine hydroxymethyltransferase (SHMT) was found in the genome of MP2. This 224 enzyme catalyzes the addition of formaldehyde to glycine, a key step for the production 225 of serine (Jiang et al., 2014), and appears to be absent in the other Lactobacillus 226 genomes analyzed in this study. Its presence in L. kunkeei MP2 could be part of specific 227 adaptation mechanisms of this species to its environment. 
229 Prophage insertions

230 Previous work in other Lactobacillus species, reported the presence of regions with

231 prophage genes in their genomes, including species such as L. rhamnosus, L. gasseri,

232 L. salivarius, L. casei, L. lactis, and L. johnsonii (Ventura et al., 2004; 2006; Kankainen

233 et al., 2009; Savabi et al., 2014; Baugher, Durmaz \& Klaenhammer, 2014). This shows

234 the widespread abundance of prophages in the genomes of Lactobacillus species, a

235 characteristic shared by L. kunkeei MP2. Two regions were identified by PHAST (Zhou

236 et al., 2011), as putative prophage insertions (Supplementary Table 2). One of them,

237 located in the region between 594,506 to 613,136 , was found to be present in all the 23

238 Lactobacillus genomes used in this work. The second region, located around 32,973 to

23975,092 , was found to be unique to $L$. kunkeei MP2, compared to other strains of $L$.

240 kunkeei, as well as other Lactobacillus species. In at least one Lactobacillus species (L.

241 gasseri), the presence of these inserted phages has been associated with the horizontal

242 transfer of genes (Baugher, Durmaz \& Klaenhammer, 2014), suggesting a possible role

243 for these elements within the genome of L. kunkeei MP2. However, the detailed

244 mechanisms, as well as the possible adaptive consequences of such events, need to be

245 explored in more detail in the future.

246

247 Comparison of L. kunkeei MP2 with other L. kunkeei strains

248 We performed a comparative genomic analysis of MP2 against sixteen publicly

249 available genome sequences of $L$. kunkeei strains (Table 1). This analysis can provide

250 us with a snapshot of the unique features that are present in this strain, based on its 
251 gene content. Recent work, highlighted the important role that genome reduction played

252 in the evolution of L. kunkeei (Tamarit et al., 2015), which suggests that we would

253 expect to find only a few genes truly unique to L. kunkeei MP2, compared to other 254 strains.

255 Pangenome analysis resulted in the identification of a set of 853 core genes (present in 256 all the strains). For the shell genes, we found that 813 genes are found between $15 \%$ to $25795 \%$ of the analyzed strains, while 1661 genes are present in less than $15 \%$ of the 258 strains (Supplementary File 1). Focusing on the MP2 strain, based on this analysis we 259 identified 113 genes that are not present in any of the other strains, representing close 260 to $7.7 \%$ of the total number of genes.

261 Using the pangenome matrix, we can visualize the relationships between the genomes 262 based only on the clustering of the proteins (presence or absence of a gene in a protein 263 group). Visualizations of the results (Figure 2), show that the strains LAni, LAce, LAan 264 and EFB6, cluster in the same group as MP2, based on their gene content. This 265 correlates with the phylogenetic placement of MP2 within the same group, based on a 266 concatenated alignment of the core genome (853 genes; 807,585 nucleotides) (Figure 267 3). The closest strain on the tree is EFB6, which was also isolated from the gut of $A$. 268 mellifera (although from a larvae affected by European foulbrood) (Djukic et al., 2015). 269 Currently there is no evidence of association between particular strains and a particular 270 species of Apis (Tamarit et al., 2015). A better understanding of the interactions 271 between the gut microbiota and the host needs to incorporate additional variables, such 272 as the surrounding environment (including flowers and fruit), seasonal variations, 
273 among other possible elements. These factors could play a strong role in the

274 association between an Apis species and a L. kunkeei strain.

275 One of the main differences of MP2 compared to the other L. kunkeei genomes, is the 276 presence of multiple phage genes inserted in several parts of the genome. One of these

277 unique phage regions can be found at coordinates $31,034-75,092$ (Figure 1). It is a 278 large region, which includes several phage-related proteins, including structural and 279 replications components. Sequence analysis using Blast, shows that these proteins are 280 related to phages that infect Gram-positive Bacteria, such as Bacillus (Hastings et al., 281 2013), Listeria (Dorscht et al., 2009), Enterococcus (Yasmin et al., 2010), and 282 Staphylococcus (Chang et al., 2013) (Supplementary Table 3).

283

284 Comparison of L. kunkeei MP2 with other Lactobacillus strains

285 Phylogenetic reconstructions using the sequence that encodes for the 16S rRNA gene, 286 shows that the closes species to L. kunkeei MP2 is L. sanfranciscensis (Figure 4). This 287 placement is in agreement with previous diversity analysis performed on acidophilic 288 bacteria (McFrederick et al., 2012), relating the taxonomy of both species.

289 Whole genome comparisons between L. kunkeei MP2 and other Lactobacillus species, 290 discovered several unique genes. One example is gtfC, which encodes for a 291 glucosyltransferase, which has been extensively studied in Streptococcus mutans, 292 where is expressed in the presence of carbohydrates such as sucrose, D-glucose, D293 fructose, among others (Shemesh et al., 2006). GtfC (as well as GftB), is also 294 considered a virulence factor in S. mutants, promoting bacterial adhesion to smooth 295 surfaces and cells (Tsumori \& Kuramitsu, 1997). Also, GtfC is part of the synthesis route 
296 of a mixture of insoluble and soluble glucans, which are important components of

297 cariogenic biofilms (Yousefi et al., 2012). Considering the rich carbohydrate

298 environment where L. kunkeei can survive, the presence of unique glucosyltransferase

299 genes, such as gftC, could facilitate bacterial colonization of flowers, as well as the 300 honey bee gut.

301 Another unique gene found in $L$. kunkeei, encodes for the adapter protein MecA, a 302 pleiotropic regulator of bacterial development. This protein has been shown to affect 303 competence, protein degradation and sporulation in Bacteria, such as Bacillus subtilis 304 (Schlothauer et al., 2003). MecA interacts with the chaperone ClpC, and with the 305 transcription factor ComK, promoting the degradation of this protein during the 306 logarithmic growth phase. The degradation of ComK stops when bacteria enters to 307 stationary growth phase, where the quorum-sensing pheromone ComX promotes the 308 synthesis of ComS, which binds to MecA and prevents the interaction of MecA-ComK 309 (Persuh, Mandic-Mulec \& Dubnau, 2002; Prepiak et al., 2011; Wahl et al., 2014). This 310 could have an effect on the biofilm generation capabilities of L. kunkeei MP2, but this 311 needs to be explored experimentally.

312 At least seven different Lactobacillus species have been characterized in the gut 313 microbiota of $A$. mellifera, where it has been suggested that they play different roles in 314 the stability of the host functions (Engel \& Moran, 2013). L. kunkeei MP2 appears to 315 have a unique set of genes when compared to other strains of $L$. kunkeei, as well as 316 with other species of Lactobacillus (Supplementary Table 3), which suggest unique 317 adaptation strategies of $L$. kunkeei MP2 to the gut of $A$. mellifera. 
318 We also identified a hypothetical protein with similarities to a low-molecular-weight 319 protein-tyrosine phosphatase (LMPTP), unique to the L. kunkeei MP2 genome, 320 compared to other L. kunkeei strains and other Lactobacillus species. This LMPTP is 321 similar to the YfkJ protein from Bacillus subtilis, which has been involved in the 322 response to ethanol stress (Musumeci et al., 2005). Ethanol, and other organic 323 compounds, are commonly present in the environment, and accumulate in the bacterial 324 membrane affecting its physical-chemical properties, and in consequence, their 325 functions (Weber \& de Bont, 1996). This could suggest a better tolerance to organic 326 compounds, such as ethanol, for L. kunkeei MP2, which could help this organism to 327 tolerate unfavorable conditions, and have a unique competitive advantage compared to 328 other Lactobacillus species (de Guchte et al., 2002).

329 The diversity of Firmicutes species in A. mellifera could imply a metabolic diversity that 330 could be crucial for honey bee fitness (Engel \& Moran, 2013). Comparative genomics of 331 Lactobacillus genomes, have shown that close to $45 \%$ of its accessory genome encode 332 for proteins involved in carbohydrate metabolism and transport functions (Ellegaard et 333 al., 2015). With this metabolic diversity found in the accessory genome, is no surprising 334 to find unique genes in the accessory genome of L. kunkeei MP2, when compared to 335 other strains of L. kunkeei, as well as other Lactobacillus species (Supplementary Table 336 3). These genes encode for proteins that take part of the degradation of carbohydrates, 337 transport of molecules, transcription, as well as membrane proteins. It is very likely that 338 some of these genes were acquired via horizontal gene transfer from a diverse group of 339 organisms, including those that inhabit the gut of $A$. mellifera. This has been observed 340 in the adaptation of strains of Gilliamela apicola and Snodgrassella alvi to the guts of 
341 the honey bee and the bumble bee (Kwong et al., 2014), as well as in other mammalian

342 guts (Shterzer \& Mizrahi, 2015).

343 Integrative and conjugative elements in MP2

344 Multiple mobile genetic elements (MGEs), were identified in the genome of $L$. kunkeei 345 MP2, including prophages, transposons, and integrases. Several of these genes were 346 unique to the MP2 genome, compared to the other draft genomes of L. kunkeei and 347 other Lactobacillus strains. To explore a possible association between MGEs and the 348 unique genes found in the genome of L. kunkeei MP2, we performed a prediction of 349 genomic islands using Island Viewer 3 (Dhillon et al., 2015). With this approach, we 350 found that most of the unique genes are found outside genomic islands (Figure 1, 351 Supplementary Table 3). This could suggest either events of gene loss or ancestral 352 transfer events in the genome of L. kunkeei MP2 (Tamarit et al., 2015).

353 Most of the MGEs found in the genome, had similarities to integrative and conjugative 354 elements (ICEs), which are characterized by their prophage-like mode of maintenance 355 (Burrus et al., 2002). To contrast this result, the uniquely identified genes in the genome 356 of L. kunkeei MP2 were compared against the ICEberg database (Bi et al., 2012) 357 (Supplementary Table 3). ICEs commonly encode for genes that provide an increased 358 fitness to the host, such as antibiotic resistance genes, phage resistance, and heavy 359 metal transport (Burrus et al., 2002). In the case of MP2 we found genes that have 360 similarities to transmembrane proteins, phage-related proteins, and antibiotic resistance 361 mechanisms, suggesting that the incorporation and stability of these unique genes in 362 the genome of L. kunkeei MP2, is providing an increase in the fitness of this bacterial 363 strain in the gut of $A$. mellifera. Among the predicted phage-like sequences, we found 
364 one coding for a $\operatorname{mef}(A) / m s r(D)$ resistance protein, with similarity to a sequence from

365 Streptococcus pyogenes, involved in the resistance to macrolides (lannelli et al., 2014).

366 In the European Union, the usage of antibiotics, and antibiotic-containing compounds, is

367 not permitted. However, macrolides (such as tylosin and streptomycin), are still used as

368 a preventive treatment against Paenibacillus larvae, the causal agent of American

369 foulbrood, in many countries (Reynaldi et al., 2010; Gaudin, Hedou \& Verdon, 2012).

370 Thus, if bees were exposed to antibiotics in their diet, it is possible that the gut

371 microbiota may have acquired the necessary molecular mechanisms to adapt and

372 survive in an exposed environment (Tarapoulouzi et al., 2013). This acquisition can be

373 explained by horizontal gene transfer events from the surrounding natural environment

374 (such as soil). Here we can find multiple bacterial and fungal species that commonly

375 produce antimicrobial compounds, and could act as a source of these resistance genes

376 (Alippi, León \& López, 2014).

377 Most of ICEs coding genes are usually present within genomic islands in the host 378 genome (Hacker \& Carniel, 2001; Boyd, Almagro-Moreno \& Parent, 2009), but in the 379 case of L. kunkeei MP2, none of the predicted ICEs genes were found in the context of 380 genomic islands according to the predictions performed with IslandViewer 3 (Dhillon et 381 al., 2015). This could suggest the presence of previously uncharacterized ICEs, or also 382 our current limitation in the detection of ICEs from Lactobacillus species.

384 Prediction of horizontal gene transfer events

385 To predict horizontally transferred genes we used Darkhorse (Podell \& Gaasterland, 386 2007) to analyze the complete genome of L. kunkeei MP2. We did not consider hits to 
387 organisms within the same Phylum, to avoid false predictions, although this could lead

388 to ignore real transfer events between more closely related organisms. A total of 19 389 genes were predicted to have been acquired via horizontal gene transfer 390 (Supplementary Table 3), with a normalized LPI score cutoff of 0.546 . Seven of these 391 genes had matches with the genome of $A$. mellifera, which a detailed look suggested as 392 a contamination of the genome of $A$. mellifera with sequences from Lactobacillus 393 species. This has been previously reported for other genome projects (Merchant, Wood 394 \& Salzberg, 2014). Only one of the genes predicted to be acquired via HGT was unique 395 to L. kunkeei MP2 when compared to other L. kunkeei strains and other Lactobacillus 396 genomes, which codifies for a hypothetical protein, with a best hit as a phage protein 397 from Halomonas sp. HAL1. None of the predicted genes was found associated with an 398 ICE or a genomic island. Although the apparent lack of genes of acquired via HGT 399 could be explained by the genome reduction that has been observed in L. kunkeei 400 strains (Tamarit et al., 2015), an alternative explanation is the lack of reference 401 genomes of isolates obtained from the gut of $A$. mellifera. This could limit our ability to 402 predict events of horizontal gene transfer between members of the honey gut 403 microbiota.

405 Conclusions

406 Using long-read sequence technologies we were able to assemble the first closed 407 genome for a $L$. kunkeei strain, resolving some of the assemble issues of a previous 408 draft of this strain (Olmos et al., 2014). The comparison of the genome sequence 409 against other Lactobacilus species, showed a percentage of genes that are unique to 
410 the MP2 strain, including metabolic key enzymes that could play an important role in the

411 honey bee nutrition and fitness. The genome of $L$. kunkeei MP2 also has genes

412 encoding for proteins involved in important roles such as adhesion, biofilm synthesis,

413 and stress tolerance, which in addition to the presence of antibiotic resistance related

414 genes, highlights the versatility of this bacteria to adapt to different environments, such

415 as flowers or insect guts.

416 One of the features highlighted in this study, is the abundance of prophages in the $L$.

417 kunkeei genome. The presence of prophages in Lactobacillus is common, but MP2 has

418 sequences unique to this strain. This is the case of a large genomic region (located in

419 the 31,034-75,092 region), with genes encoding for several phage-related proteins,

420 including structural and replicative components. The presence of prophages could be

421 associated with lateral transference events, and therefore, with the acquisition of genes

422 related with bacterial fitness. Given the high percentage of hypothetical proteins

423 encoded in this region, a future goal for research, is the elucidation of the role for these

424 proteins in L. kunkeei MP2.

\section{References}

426

427

428

429

430

431

432

433

434

435

436

437

438

439
Alippi AM, León IE, López AC 2014. Tetracycline-resistance encoding plasmids from Paenibacillus larvae, the causal agent of American foulbrood disease, isolated from commercial honeys. International microbiology : the official journal of the Spanish Society for Microbiology 17:49-61.

Anderson KE, Sheehan TH, Mott BM, Maes P, Snyder L, Schwan MR, Walton A, Jones BM, Corby-Harris V 2013. Microbial ecology of the hive and pollination landscape: bacterial associates from floral nectar, the alimentary tract and stored food of honey bees (Apis mellifera). PLoS ONE 8:e83125.

Audisio MC, Benítez-Ahrendts MR 2011. Lactobacillus johnsonii CRL1647, isolated from Apis mellifera L. bee-gut, exhibited a beneficial effect on honeybee colonies. Beneficial microbes 2:29-34.

Audisio MC, Sabaté DC, Benítez-Ahrendts MR 2015. Effect of Lactobacillus johnsonii CRL1647 on different parameters of honeybee colonies and bacterial populations of the bee gut. Beneficial microbes:1-10. 
440 Bae S, Fleet GH, Heard GM 2006. Lactic acid bacteria associated with wine grapes

441

442

443

444

445

446

447

448

449

450

451

452

453

454

455

456

457

458

459

460

461

462

463

464

465

466

467

468

469

470

471

472

473

474

475

476

477

478

479

480

481

482

483

484

485 from several Australian vineyards. Journal of Applied Microbiology 100:712-727.

Baugher JL, Durmaz E, Klaenhammer TR 2014. Spontaneously induced prophages in Lactobacillus gasseri contribute to horizontal gene transfer. Applied and Environmental Microbiology 80:3508-3517.

Berlin K, Koren S, Chin C-S, Drake JP, Landolin JM, Phillippy AM 2015. Assembling large genomes with single-molecule sequencing and locality-sensitive hashing. Nature Biotechnology 33:623-630.

Bi D, Xu Z, Harrison EM, Tai C, Wei Y, He X, Jia S, Deng Z, Rajakumar K, Ou H-Y 2012. ICEberg: a web-based resource for integrative and conjugative elements found in Bacteria. Nucleic Acids Research 40:D621-6.

Boom R, Sol C, Beld M, Weel J, Goudsmit J, Wertheim-van Dillen P 1999. Improved silica-guanidiniumthiocyanate DNA isolation procedure based on selective binding of bovine alpha-casein to silica particles. Journal of clinical microbiology 37:615619.

Boyd EF, Almagro-Moreno S, Parent MA 2009. Genomic islands are dynamic, ancient integrative elements in bacterial evolution. Trends in Microbiology 17:47-53.

Burrus V, Pavlovic G, Decaris B, Guédon G 2002. Conjugative transposons: the tip of the iceberg. Molecular Microbiology 46:601-610.

Chang Y, Lee J-H, Shin H, Heu S, Ryu S 2013. Characterization and complete genome sequence analysis of Staphylococcus aureus bacteriophage SA12. Virus genes 47:389-393.

Corby-Harris V, Maes P, Anderson KE 2014. The bacterial communities associated with honey bee (Apis mellifera) foragers. PLoS ONE 9:e95056.

Cox-Foster DL, Conlan S, Holmes EC, Palacios G, Evans JD, Moran NA, Quan P-L, Briese T, Hornig M, Geiser DM, Martinson V, vanEngelsdorp D, Kalkstein AL, Drysdale A, Hui J, Zhai J, Cui L, Hutchison SK, Simons JF, Egholm M, Pettis JS, Lipkin WI 2007. A metagenomic survey of microbes in honey bee colony collapse disorder. Science 318:283-287.

Daum M, Herrmann S, Wilkinson B, Bechthold A 2009. Genes and enzymes involved in bacterial isoprenoid biosynthesis. Current Opinion in Chemical Biology 13:180-188.

de Guchte van M, Serror P, Chervaux C, Smokvina T, Ehrlich SD, Maguin E 2002. Stress responses in lactic acid bacteria. Antonie van Leeuwenhoek 82:187-216.

Dhillon BK, Laird MR, Shay JA, Winsor GL, Lo R, Nizam F, Pereira SK, Waglechner N, McArthur AG, Langille MGI, Brinkman FSL 2015. IslandViewer 3: more flexible, interactive genomic island discovery, visualization and analysis. Nucleic Acids Research 43:W104-8.

Di Pasquale G, Salignon M, Le Conte Y, Belzunces LP, Decourtye A, Kretzschmar A, Suchail S, Brunet J-L, Alaux C 2013. Influence of pollen nutrition on honey bee health: do pollen quality and diversity matter? PLOS ONE 8:e72016.

Di Prisco G, Cavaliere V, Annoscia D, Varricchio P, Caprio E, Nazzi F, Gargiulo G, Pennacchio F 2013. Neonicotinoid clothianidin adversely affects insect immunity and promotes replication of a viral pathogen in honey bees. Proceedings of the National Academy of Sciences 110:18466-18471.

Djukic M, Poehlein A, Strauß J, Tann FJ, Leimbach A, Hoppert M, Daniel R 2015. High quality draft genome of Lactobacillus kunkeei EFB6, isolated from a German 
486

487

488

489

490

491

492

493

494

495

496

497

498

499

500

501

502

503

504

505

506

507

508

509

510

511

512

513

514

515

516

517

518

519

520

521

522

523

524

525

526

527

528

529

530

531

European foulbrood outbreak of honeybees. Standards in Genomic Sciences 10:16. Dorscht J, Klumpp J, Bielmann R, Schmelcher M, Born Y, Zimmer M, Calendar R, Loessner MJ 2009. Comparative genome analysis of Listeria bacteriophages reveals extensive mosaicism, programmed translational frameshifting, and a novel prophage insertion site. Journal Of Bacteriology 191:7206-7215.

Edwards CG, Haag KM, Collins MD, Hutson RA, Huang YC 1998. Lactobacillus kunkeei sp. nov.: a spoilage organism associated with grape juice fermentations. Journal of Applied Microbiology 84:698-702.

Ellegaard KM, Tamarit D, Javelind E, Olofsson TC, Andersson SGE, Vásquez A 2015. Extensive intra-phylotype diversity in lactobacilli and bifidobacteria from the honeybee gut. BMC Genomics 16:284.

Endo A 2012. Fructophilic lactic acid bacteria inhabit fructose-rich niches in nature. Microbial ecology in health and disease 23:647.

Endo A, Salminen S 2013. Honeybees and beehives are rich sources for fructophilic lactic acid bacteria. Systematic and Applied Microbiology 36:444-448.

Endo A, Futagawa-Endo Y, Dicks LMT 2009. Isolation and characterization of fructophilic lactic acid bacteria from fructose-rich niches. Systematic and Applied Microbiology 32:593-600.

Endo A, Irisawa T, Futagawa-Endo Y, Takano K, Toit du M, Okada S, Dicks LMT 2012. Characterization and emended description of Lactobacillus kunkeei as a fructophilic lactic acid bacterium. International Journal of Systematic And Evolutionary Microbiology 62:500-504.

Engel P, Moran NA 2013. Functional and evolutionary insights into the simple yet specific gut microbiota of the honey bee from metagenomic analysis. Gut microbes 4:60-65.

Eren AM, Esen ÖC, Quince C, Vineis JH, Morrison HG, Sogin ML, Delmont TO 2015. Anvi'o: an advanced analysis and visualization platform for 'omics data. PeerJ 3:e1319.

Evans JD, Schwarz RS 2011. Bees brought to their knees: microbes affecting honey bee health. Trends in Microbiology 19:614-620.

Gaudin V, Hedou C, Verdon E 2012. Validation of two ELISA kits for the screening of tylosin and streptomycin in honey according to the European decision 2002/657/EC. Food Additives \& Contaminants: Part A 30:93-109.

Hacker J, Carniel E 2001. Ecological fitness, genomic islands and bacterial pathogenicity. A Darwinian view of the evolution of microbes. EMBO reports 2:376381.

Hastings WJ, Ritter MA, Chamakura KR, Kuty Everett GF 2013. Complete Genome of Bacillus megaterium Siphophage Staley. Genome announcements 1:e00864-13e00864-13.

Henry M, Béguin M, Requier F, Rollin O, Odoux J-F, Aupinel P, Aptel J, Tchamitchian $S$, Decourtye A 2012. A common pesticide decreases foraging success and survival in honey bees. Science 336:348-350.

lannelli F, Santagati M, Santoro F, Oggioni MR, Stefani S, Pozzi G 2014. Nucleotide sequence of conjugative prophage $\$ 1207.3$ (formerly Tn1207.3) carrying the $\operatorname{mef}(A) / m s r(D)$ genes for efflux resistance to macrolides in Streptococcus pyogenes. Frontiers in Microbiology 5:687. 
532 Jiang W, Chen L, Hu N, Yuan S, Li B, Liu Z 2014. A novel serine

533

534

535

536

537

538

539

540

541

542

543

544

545

546

547

548

549

550

551

552

553

554

555

556

557

558

559

560

561

562

563

564

565

566

567

568

569

570

571

572

573

574

575

576

577

hydroxymethyltransferase from Arthrobacter nicotianae: characterization and improving catalytic efficiency by rational design. BMC biotechnology 14:93.

Kankainen M, Paulin L, Tynkkynen S, Ossowski von I, Reunanen J, Partanen P, Satokari R, Vesterlund S, Hendrickx APA, Lebeer S, De Keersmaecker SCJ, Vanderleyden J, Hämäläinen T, Laukkanen S, Salovuori N, Ritari J, Alatalo E, Korpela R, Mattila-Sandholm T, Lassig A, Hatakka K, Kinnunen KT, Karjalainen H, Saxelin M, Laakso K, Surakka A, Palva A, Salusjärvi T, Auvinen P, de Vos WM 2009. Comparative genomic analysis of Lactobacillus rhamnosus GG reveals pili containing a human- mucus binding protein. Proceedings of the National Academy of Sciences 106:17193-17198.

Krzywinski M, Schein J, Birol I, Connors J, Gascoyne R, Horsman D, Jones SJ, Marra MA 2009. Circos: An information aesthetic for comparative genomics. Genome Research 19:1639-1645.

Kurtz S, Phillippy A, Delcher AL, Smoot M, Shumway M, Antonescu C, Salzberg SL 2004. Versatile and open software for comparing large genomes. Genome Biology 5:R12.

Kwong WK, Engel P, Koch H, Moran NA 2014. Genomics and host specialization of honey bee and bumble bee gut symbionts. Proceedings of the National Academy of Sciences 111:11509-11514.

Lozo J, Begovic J, Jovcic B, Golic N, Topisirovic L 2008. Effect of Methionine and Cysteine Deprivation on Growth of Different Natural Isolates of Lactobacillus Spp. in Chemically Defined Media. Archives of Biological Sciences 60:509-517.

Löytynoja A 2014. Phylogeny-aware alignment with PRANK. Methods in molecular biology (Clifton, N.J.) 1079:155-170.

Martinson VG, Danforth BN, Minckley RL, Rueppell O, Tingek S, Moran NA 2011. A simple and distinctive microbiota associated with honey bees and bumble bees. Molecular Ecology 20:619-628.

McFrederick QS, Wcislo WT, Hout MC, Mueller UG 2014. Host species and developmental stage, but not host social structure, affects bacterial community structure in socially polymorphic bees. FEMS Microbiology Ecology 88:398-406.

McFrederick QS, Wcislo WT, TAYLOR DR, ISHAK HD, DOWD SE, Mueller UG 2012. Environment or kin: whence do bees obtain acidophilic bacteria? Molecular Ecology 21:1754-1768.

Merchant S, Wood DE, Salzberg SL 2014. Unexpected cross-species contamination in genome sequencing projects. PeerJ 2:e675.

Musumeci L, Bongiorni C, Tautz L, Edwards RA, Osterman A, Perego M, Mustelin T, Bottini N 2005. Low-molecular-weight protein tyrosine phosphatases of Bacillus subtilis. Journal Of Bacteriology 187:4945-4956.

Myers EW, Sutton GG, Delcher AL, Dew IM, Fasulo DP, Flanigan MJ, Kravitz SA, Mobarry CM, Reinert KH, Remington KA, Anson EL, Bolanos RA, Chou HH, Jordan CM, Halpern AL, Lonardi S, Beasley EM, Brandon RC, Chen L, Dunn PJ, Lai Z, Liang Y, Nusskern DR, Zhan M, Zhang Q, Zheng X, Rubin GM, Adams MD, Venter JC 2000. A whole-genome assembly of Drosophila. Science 287:2196-2204.

Neveling DP, Endo A, Dicks LMT 2012. Fructophilic Lactobacillus kunkeei and Lactobacillus brevis isolated from fresh flowers, bees and bee-hives. Current 
Olmos A, Henríquez-Piskulich P, Sanchez C, Rojas-Herrera M, Moreno-Pino M, Gómez M, Rodríguez Da Silva R, Maracaja-Coutinho V, Aldea P, Trombert AN 2014. Draft

581

582

583

584

585

586

587

588

589

590

591

592

593

594

595

596

597

598

599

600

601

602

603

604

605

606

607

608

609

610

611

612

613

614

615

616

617

618

619

620

621

622

623 Genome of Chilean Honeybee (Apis mellifera) Gut Strain Lactobacillus kunkeei MP2. Genome announcements 2:e01013-14-e01013-14.

Page AJ, Cummins CA, Hunt M, Wong VK, Reuter S, Holden MTG, Fookes M, Falush D, Keane JA, Parkhill J 2015. Roary: Rapid large-scale prokaryote pan genome analysis. Bioinformatics (Oxford, England):btv421.

Persuh M, Mandic-Mulec I, Dubnau D 2002. A MecA paralog, YpbH, binds ClpC, affecting both competence and sporulation. Journal Of Bacteriology 184:2310-2313.

Podell S, Gaasterland T 2007. DarkHorse: a method for genome-wide prediction of horizontal gene transfer. Genome Biology 8:R16.

Porcellato D, Frantzen C, Rangberg A, Umu OC, Gabrielsen C, Nes IF, Amdam GV, Diep DB 2015. Draft Genome Sequence of Lactobacillus kunkeei AR114 Isolated from Honey Bee Gut. Genome announcements 3:e00144-15.

Powell S, Forslund K, Szklarczyk D, Trachana K, Roth A, Huerta-Cepas J, Gabaldón T, Rattei T, Creevey C, Kuhn M, Jensen LJ, Mering von C, Bork P 2014. eggNOG v4.0: nested orthology inference across 3686 organisms. Nucleic Acids Research 42:D231-D239.

Prepiak P, Defrancesco M, Spadavecchia S, Mirouze N, Albano M, Persuh M, Fujita M, Dubnau D 2011. MecA dampens transitions to spore, biofilm exopolysaccharide and competence expression by two different mechanisms. Molecular Microbiology 80:1014-1030.

Price MN, Dehal PS, Arkin AP 2010. FastTree 2--approximately maximum-likelihood trees for large alignments. PLOS ONE 5:e9490.

Pruesse E, Peplies J, Glöckner FO 2012. SINA: accurate high-throughput multiple sequence alignment of ribosomal RNA genes. Bioinformatics (Oxford, England) 28:1823-1829.

Quast C, Pruesse E, Yilmaz P, Gerken J, Schweer T, Yarza P, Peplies J, Glöckner FO 2013. The SILVA ribosomal RNA gene database project: improved data processing and web-based tools. Nucleic Acids Research 41:D590-6.

Reynaldi FJ, Lacunza J, Alippi AM, Rule R 2010. Unión de los antibióticos tilosina, tilmicosina y oxitetraciclina a proteínas presentes en abejas, larvas y productos de la colmena de Apis mellifera L. Revista argentina de microbiología 42:279-283.

Savabi O, Kazemi M, Kamali S, Salehi AR, Eslami G, Tahmourespour A, Salehi R 2014. Effects of biosurfactant produced by Lactobacillus casei on gtfB, gtfC, and ftf gene expression level in S. mutans by real-time RT-PCR. Advanced biomedical research 3:231.

Schlothauer T, Mogk A, Dougan DA, Bukau B, Turgay K 2003. MecA, an adaptor protein necessary for ClpC chaperone activity. Proceedings of the National Academy of Sciences of the United States of America 100:2306-2311.

Seefeldt KE, Weimer BC 2000. Diversity of sulfur compound production in lactic acid bacteria. Journal of dairy science 83:2740-2746.

Seemann T 2014. Prokka: rapid prokaryotic genome annotation. Bioinformatics (Oxford, England) 30:2068-2069.

Shemesh M, Tam A, Feldman M, Steinberg D 2006. Differential expression profiles of 
624

625

626

627

628

629

630

631

632

633

634

635

636

637

638

639

640

641

642

643

644

645

646

647

648

649

650

651

652

653

654

655

656

657

658

659

660

661

662

663

664

665

666

667

668

669

Streptococcus mutans ftf, gtf and vicR genes in the presence of dietary carbohydrates at early and late exponential growth phases. Carbohydrate research 341:2090-2097.

Shterzer N, Mizrahi I 2015. The animal gut as a melting pot for horizontal gene transfer. Canadian Journal of Microbiology:1-3.

Sloan DB, Moran NA 2012. Endosymbiotic bacteria as a source of carotenoids in whiteflies. Biology Letters 8:986-989.

Tamarit D, Ellegaard KM, Wikander J, Olofsson T, Vásquez A, Andersson SGE 2015. Functionally Structured Genomes in Lactobacillus kunkeei Colonizing the Honey Crop and Food Products of Honeybees and Stingless Bees. Genome Biology and Evolution 7:1455-1473.

Tarapoulouzi M, Papachrysostomou C, Constantinou S, Kanari P, Hadjigeorgiou M 2013. Determinative and confirmatory method for residues of tetracyclines in honey by LC-MS/MS. Food additives \& contaminants. Part A, Chemistry, analysis, control, exposure \& risk assessment 30:1728-1732.

Tsumori H, Kuramitsu H 1997. The role of the Streptococcus mutans glucosyltransferases in the sucrose-dependent attachment to smooth surfaces: essential role of the GtfC enzyme. Oral microbiology and immunology 12:274-280.

Vásquez A, Forsgren E, Fries I, Paxton RJ, Flaberg E, Szekely L, Olofsson TC 2012. Symbionts as major modulators of insect health: lactic acid bacteria and honeybees. PLOS ONE 7:e33188.

Ventura M, Canchaya C, Bernini V, Altermann E, Barrangou R, McGrath S, Claesson MJ, Li Y, Leahy S, Walker CD, Zink R, Neviani E, Steele J, Broadbent J, Klaenhammer TR, Fitzgerald GF, O'Toole PW, van Sinderen D 2006. Comparative genomics and transcriptional analysis of prophages identified in the genomes of Lactobacillus gasseri, Lactobacillus salivarius, and Lactobacillus casei. Applied and Environmental Microbiology 72:3130-3146.

Ventura M, Canchaya C, Pridmore RD, Brüssow H 2004. The prophages of Lactobacillus johnsonii NCC 533: comparative genomics and transcription analysis. Virology 320:229-242.

Wahl A, Servais F, Drucbert A-S, Foulon C, Fontaine L, Hols P 2014. Control of natural transformation in salivarius Streptococci through specific degradation of $\sigma \mathrm{X}$ by the MecA-ClpCP protease complex. Journal Of Bacteriology 196:2807-2816.

Weber FJ, de Bont JA 1996. Adaptation mechanisms of microorganisms to the toxic effects of organic solvents on membranes. Biochimica et biophysica acta 1286:225245.

Yasmin A, Kenny JG, Shankar J, Darby AC, Hall N, Edwards C, Horsburgh MJ 2010. Comparative genomics and transduction potential of Enterococcus faecalis temperate bacteriophages. Journal Of Bacteriology 192:1122-1130.

Yousefi B, Ghaderi S, Rezapoor-Lactooyi A, Amiri N, Verdi J, Shoae-Hassani A 2012. Hydroxy decenoic acid down regulates gtfB and gtfC expression and prevents Streptococcus mutans adherence to the cell surfaces. Annals of clinical microbiology and antimicrobials 11:21.

Zhou Y, Liang Y, Lynch KH, Dennis JJ, Wishart DS 2011. PHAST: A Fast Phage Search Tool. Nucleic Acids Research 39:W347-W352. 
Figure 1 (on next page)

Genome organisation of L. kunkeei MP2

Circular overview of the complete genome of L. kunkeei MP2, highlighthing some of the features. Starting from the outside ring towards the interior: EggNOG annotation of the predicted CDS; Contig recruitment of the previous L. kunkeei MP2 genome sequencing (Olmos et al., 2014); Phage island predictions using Island Viewer 3; Unique genes of $L$. kunkeei MP2, compared to 16 strains of L. kunkeei; Unique genes of L. kunkeei MP2 compared with 22 genomes of Lactobacillus species; \%GC contento of the L. kunkeei MP2 genome 


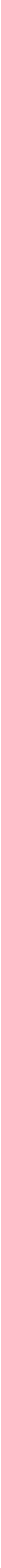


Figure 2 (on next page)

Anvi'o pangenome visualization of 16 L. kunkeei genomes

The outer core in red, shows the core genome of L. kunkeei, protein clusters shared among all the strains (853 genes). The genomes are clustered based on the presence/absence pattern of protein clusters. MP2 is highlighted in green, while the more similar strains based on the clustering pattern, are highlighted in lighter green (LAni, LAce, LAan, and EFB6). Pangenome visualization was generated using Anvi'o (Eren et al. 2015). 


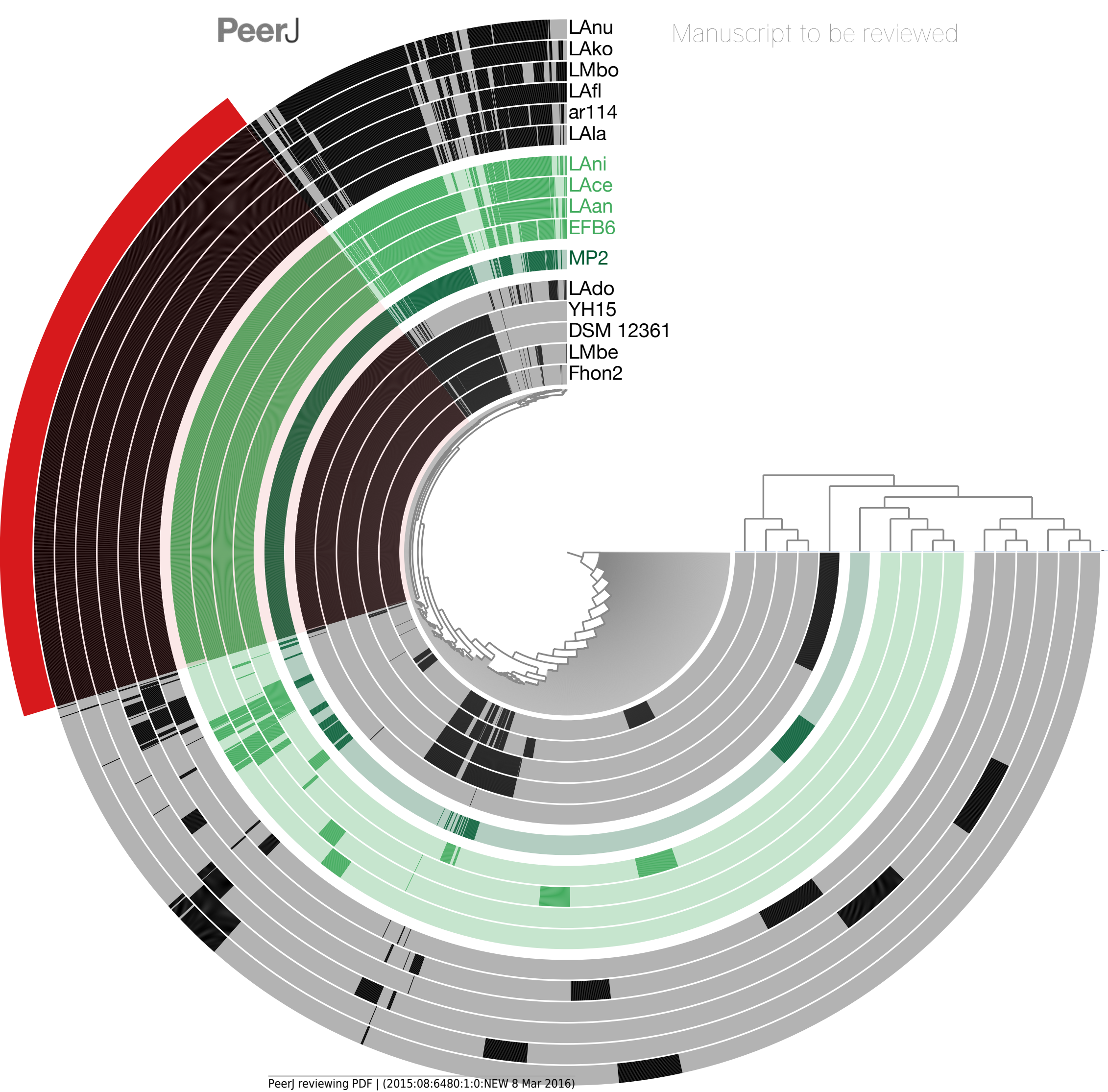


Figure 3 (on next page)

Phylogenetic tree of 16 L. kunkeei strains

The tree was constructed using all of the genes shared between all 16 strains (853 genes; 807,585 nucleotides). 


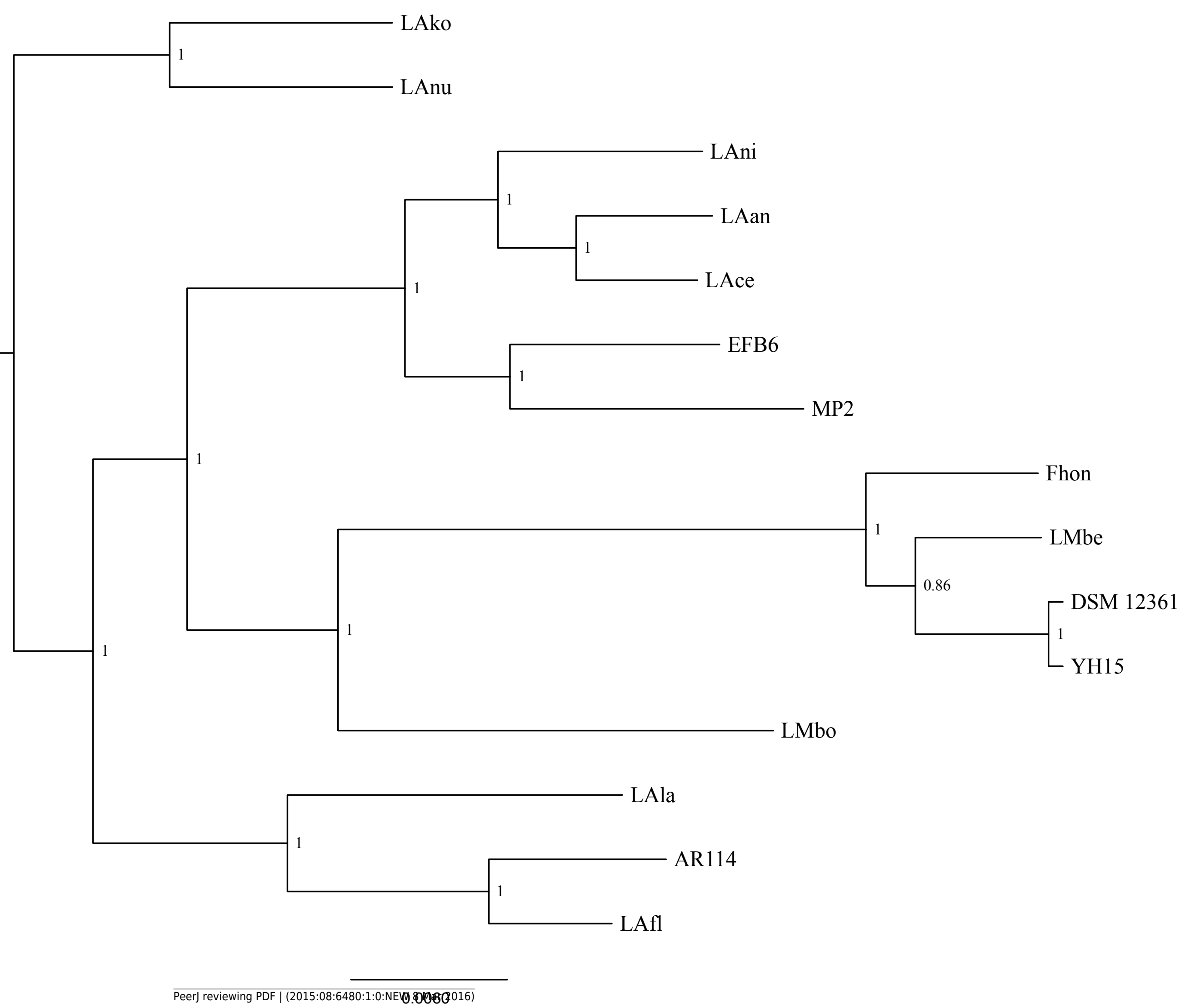


Figure 4 (on next page)

Phylogenetic tree of several Lactobacillus species, including L. kunkeei MP2

Phylogenetic reconstruction was done using the sequence of the 16S rRNA gene. 


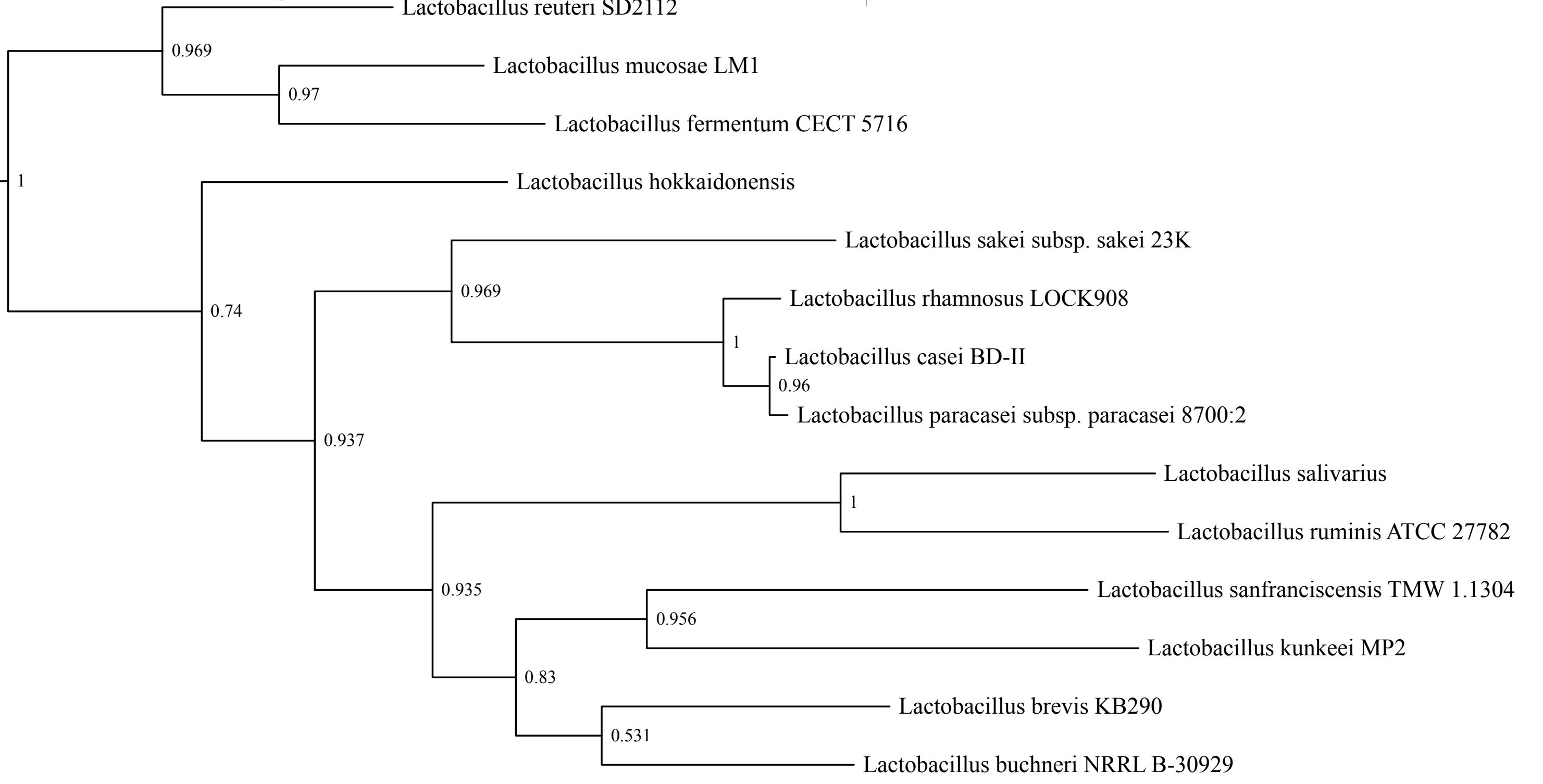

Lactobacillus johnsonii DPC 6026 0.994

- Lactobacillus gasseri 130918

Lactobacillus delbrueckii subsp. bulgaricus ND02 0.999

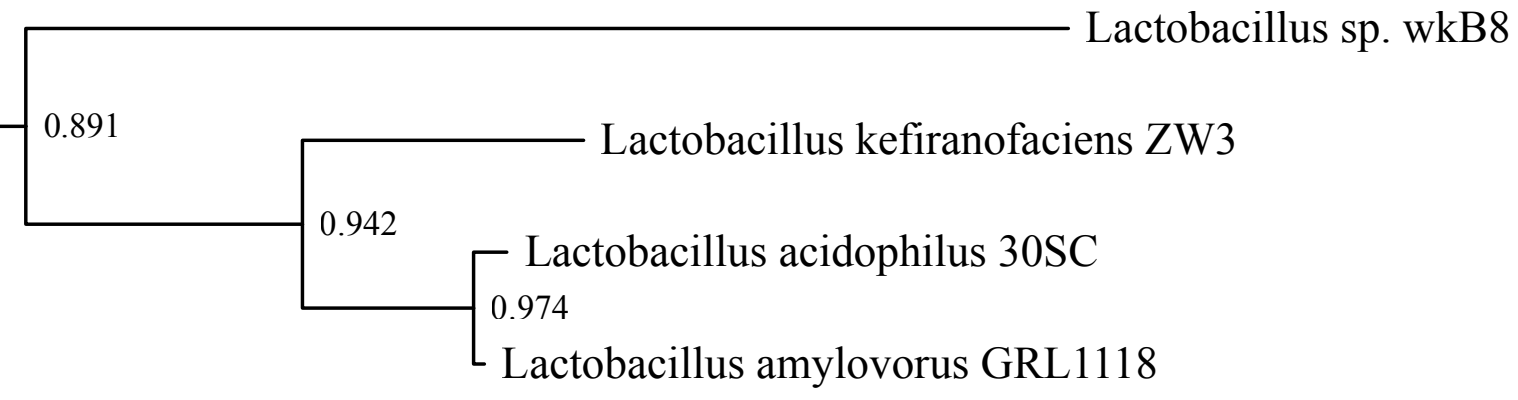




\section{Table 1 (on next page)}

Genome sequences used in this study

Species name and accession numbers of the genomes selected in this study 


\begin{tabular}{|c|c|c|}
\hline Genomes & Bioproject & Assembly \\
\hline Lactobacillus kunkeei DSM 12361 & PRJNA222257 & GCA_001433825.1 \\
\hline Lactobacillus kunkeei Fhon2 & PRJNA270967 & GCA_001281165.1 \\
\hline Lactobacillus kunkeei LAan & PRJNA270961 & GCA_001281225.1 \\
\hline Lactobacillus kunkeei LAce & PRJNA270962 & GCA_001421115.1 \\
\hline Lactobacillus kunkeei LAfl & PRJNA270964 & GCA_001421135.1 \\
\hline Lactobacillus kunkeei LAko & PRJNA270965 & GCA_001281205.1 \\
\hline Lactobacillus kunkeei LAla & PRJNA270966 & GCA_001281215.1 \\
\hline Lactobacillus kunkeei LAni & PRJNA270969 & GCA_001281285.1 \\
\hline Lactobacillus kunkeei LMbe & PRJNA270972 & GCA_001308185.1 \\
\hline Lactobacillus kunkeei LMbo & PRJNA270973 & GCA_001308195.1 \\
\hline Lactobacillus kunkeei LAdo & PRJNA270963 & GCA_001308205.1 \\
\hline Lactobacillus kunkeei LAnu & PRJNA270970 & GCA_001308215.1 \\
\hline Lactobacillus kunkeei EFB6 & PRJNA227106 & GCA_000687335.1 \\
\hline Lactobacillus kunkeei AR114 & PRJNA253911 & GCA_000830375.1 \\
\hline Lactobacillus kunkeei $\mathrm{YH}-15$ & PRJNA270974 & GCA_001281265.1 \\
\hline Lactobacillus kunkeei MP2 & PRJNA298292 & GCA_001314945.1 \\
\hline Lactobacillus acidophilus 30SC & PRJNA63605 & GCA_000191545.1 \\
\hline Lactobacillus amylovorus GRL1118 & PRJNA160233 & GCA_000194115.1 \\
\hline Lactobacillus brevis KB290 & PRJNA195560 & GCA_000359625.1 \\
\hline Lactobacillus buchneri NRRL B-30929 & PRJNA66205 & GCA_000211375.1 \\
\hline Lactobacillus casei BD-II & PRJNA162119 & GCA_000194765.1 \\
\hline Lactobacillus delbrueckii subsp. bulgaricus ND02 & PRJNA60621 & GCA_000182835.1 \\
\hline Lactobacillus fermentum CECT 5716 & PRJNA162003 & GCA_000210515.1 \\
\hline Lactobacillus gasseri 130918 & PRJNA224116 & GCA_000814885.1 \\
\hline Lactobacillus helveticus $\mathrm{H} 10$ & PRJNA162017 & GCA_000189515.1 \\
\hline Lactobacillus hokkaidonensis JCM 18461 & PRJNA224116 & GCA_000829395.1 \\
\hline Lactobacillus johnsonii DPC 6026 & PRJNA162057 & GCA_000204985.1 \\
\hline Lactobacillus kefiranofaciens ZW3 & PRJNA67985 & GCA_000214785.1 \\
\hline Lactobacillus mucosae LM1 & PRJNA86029 & GCA_000248095.3 \\
\hline Lactobacillus paracasei subsp. paracasei $8700: 2$ & PRJNA55295 & GCA_000155515.2 \\
\hline Lactobacillus plantarum & PRJNA224116 & GCA_000931425.1 \\
\hline Lactobacillus reuteri SD2112 & PRJNA55357 & GCA_000159455.2 \\
\hline Lactobacillus rhamnosus LOCK908 & PRJNA210958 & GCA_000418495.1 \\
\hline Lactobacillus ruminis ATCC 27782 & PRJNA73417 & GCA_000224985.1 \\
\hline Lactobacillus sakei subsp. sakei $23 \mathrm{~K}$ & PRJNA58281 & GCA_000026065.1 \\
\hline Lactobacillus salivarius & PRJNA224116 & GCA_000758365.1 \\
\hline Lactobacillus sanfranciscensis TMW 1.1304 & PRJNA72937 & GCA_000225325.1 \\
\hline Lactobacillus sp. wkB8 & PRJNA224116 & GCA_000761135.1 \\
\hline
\end{tabular}


1

Peer] reviewing PDF | (2015:08:6480:1:0:NEW 8 Mar 2016) 


\section{Table 2 (on next page)}

Summary of the functional assignation for the predicted genes of $L$. kunkeei MP2 based on EggNOG categories 
Information Storage and Processing

$\begin{array}{ll}\text { Translation, ribosomal structure and biogenesis } & 127\end{array}$

$\begin{array}{ll}\text { Transcription } & 73\end{array}$

$\begin{array}{ll}\text { Replication, recombination and repair } & 129\end{array}$

\section{Cellular Processes and Signaling}

Cell cycle control, cell division, chromosome partitioning 22

Defense mechanisms $\quad 15$

$\begin{array}{ll}\text { Signal transduction mechanisms } & 23\end{array}$

Cell wall/membrane/envelope biogenesis $\quad 77$

Cell motility $\quad 4$

Intracellular trafficking, secretion, and vesicular transport $\quad 19$

Posttranslational modification, protein turnover,

chaperones

\section{Metabolism}

Energy production and conversion $\quad 41$

Carbohydrate transport and metabolism $\quad 58$

$\begin{array}{ll}\text { Amino acid transport and metabolism } & 107\end{array}$

Nucleotide transport and metabolism $\quad 69$

Coenzyme transport and metabolism 25

Lipid transport and metabolism $\quad 32$

Inorganic ion transport and metabolism 65

Secondary metabolites biosynthesis, transport and

catabolism

8

\section{Poorly Characterized}

Function unknown 